\title{
ADEQUACY OF METHODOLOGY FOR CONDUCTING GERMINATION TEST IN FORAGE PEA SEEDS
}

\author{
ADEQUAÇÃO DA METODOLOGIA PARA CONDUÇÃO DO TESTE DE \\ GERMINAÇÃO EM SEMENTES DE ERVILHA FORRAGEIRA
}

\section{Carla Gomes MACHADO' ${ }^{1}$; Cibele Chalita MARTINS ${ }^{2}$; Givanildo Zildo SILVA ${ }^{3}$; Simério Carlos Silva CRUZ ${ }^{4}$; Raissa Macedo ASSIS ${ }^{5}$}

1. Engenheira Agrônoma, Professora, Doutora, Universidade Federal de Goiás - UFG, Campus Jatobá, Jataí, GO, Brasil; 2. Engenheira Agrônoma, Professora Livre-Docente, Faculdade de Ciências Agrárias e Veterinárias - UNESP, Jaboticabal, SP, Brasil; 3. Engenheiro Agrônomo, Professor, Pós-Doutorado, Universidade Federal de Goiás - UFG, Campus Jatobá, Jataí, GO, Brasil. Autor correspondente: givanildozildo@ hotmail.com; 4. Engenheiro Agrônomo, Professor, Doutor, Universidade Federal de Goiás - UFG, Campus Jatobá,

Jataí, GO, Brasil; 5. Engenheira Agrônoma, Mestranda, Universidade Federal de Goiás - UFG, Campus Jatobá, Jataí, GO, Brasil.

\begin{abstract}
Seeds presents variable germination performance at different temperatures and substrates, which are basic components of germination test. Within this context, this work aimed to adapt the methodology for the seeds germination's test of P. sativum subsp. arvense. The experiment required four portions of seeds, five temperature degrees $\left(15,20,25,20-30\right.$ and $15-25{ }^{\circ} \mathrm{C}$ ), and two substrata (roll of paper and in sand). Seeds evaluation considered the following: moisture degree and germination test. The effect of seed portion, substrata and temperature in germination was assessed on a daily basis. It was calculated the rate of germination, of anormal plants, of dormant and dead seeds, and of speed of germination index; initial, final and average times, and synchrony. The experimental design consisted of random blocks with four replications, while conjoint analysis was the statistical procedure adopted. The germination test for forage pea seeds should be conducted at the constant temperature $20{ }^{\circ} \mathrm{C}$ in roll of paper substrate with the first count and final count on the 4th and 7th day, respectively.
\end{abstract}

KEYWORDS: Pisum sativum subsp. arvense. Substratum. Temperature. Quality control. Speed of germination.

\section{INTRODUCTION}

The production of forage pea (Pisum sativum subsp. Arvense) is intended for animal feeding (GIORDANO; PEREIRA, 1989; TAN et al., 2013; CARGNELUTTI FILHO et al., 2015). In addition, this genotype presents important characteristics for green manuring and soil coverage, being cultivated in the winter, when many agricultural areas remain unused in Brazil (PINNOW et al., 2013; CARGNELUTTI FILHO et al., 2015).

For commercial $P$. sativum subsp. arvense seeds, regulated by seed standards, the requirements are lower as regards the quality of lots in relation to large vegetable crops, which is due to the fact that the technology involved in the production of seeds of this species is less developed in relation to the other species cultivated for longer time (MACHADO et al., 2011). In the laboratory, methods of analysis have been studied, aiming at the regular, rapid and complete germination of seeds under controlled conditions. Such conditions, considered to be optimal, are standardized so that the results of germination tests can be reproduced and compared, within limits tolerated by the Rules for Seed Testing - RAS (BRASIL, 2009).

For $P$. sativum subsp. arvense, there is no specific methodology for the conduction of the germination test in RAS, requiring the adequacy of the methodology aiming at the routine use in the laboratory of seed analysis. The rules recommend for pea, paper and sand substrates, temperature of 20 ${ }^{\circ} \mathrm{C}$ and evaluations at five and eight days; still recommend the use of sand replacing paper, when the evaluation of a seed sample is impractical due to contamination of the paper substrate (BRASIL, 2009). The choice of substrate is at the discretion of the analytical laboratory, depending on the availability of materials and the analyst's familiarity with the analytical method.

Within the quality control program of seed companies, germination evaluation is fundamental and necessary for successful lot production and approval for national and international market (BRASIL, 2009; TOMAZ et al., 2015; TOMAZ et al., 2016). RAS standards and procedures are periodically reviewed by a committee composed of researchers and professionals of the area, but standards and modifications for new species can 
only be added based on research results (TOMAZ et al., 2016). In this context, the aim of this study was to adapt the methodology for the germination test of forage pea seeds.

\section{MATERIAL AND METHODS}

For the determination of the methodology for conducting the germination test, four commercial lots of forage seed were used, with lots 1 and 2 of BRS cultivar, and lots 3 and 4 of IAPAR 83 cultivar. These samples were homogenized according to RAS (BRASIL, 2009), and after this procedure, seeds were conditioned in Kraft paper bags, kept in controlled environment at $5{ }^{\circ} \mathrm{C}$ until the experiment was carried out, in which they were submitted to determinations described below.

Water content - evaluated by the stove method at $105 \pm 3{ }^{\circ} \mathrm{C}$ for $24 \mathrm{~h}$ (BRASIL, 2009) using two subsamples of $5.0 \mathrm{~g}$ of seeds per lot.

Germination test - conducted with four subsamples of 100 seeds on two substrates: roll of paper towel - sheets were moistened with deionized water in the amount of 2.5 times the mass of the non-hydrated paper; and sand at two centimeters deep and moistened to $60 \%$ holding capacity, kept inside transparent plastic trays $(30.2 \times 20.8 \times 6.3$ $\mathrm{cm})$. Prior to use, sand was sieved and sterilized at $200{ }^{\circ} \mathrm{C}$ for two hours. After sowing, the boxes and the rolls were packed in plastic bags to avoid dehydration of substrates (COIMBRA et al., 2007). Normal seedlings were those whose epicotyl had surpassed the sand and when they reached one centimeter of length in the paper roll, and counts were performed until germination stabilization.

During the germination test, seeds were kept in a germination chamber under constant $(15,20$ and $25{ }^{\circ} \mathrm{C}$ ) and alternated temperature conditions (20-30 ${ }^{\circ} \mathrm{C}$ and $\left.15-25{ }^{\circ} \mathrm{C}\right)$, under a photoperiod of $8 \mathrm{~h}$ light.

In addition to the percentage of germination at the end of the test, the percentage of abnormal seedlings, dormant and dead seeds was counted. For this, the sand substrate was passed in a sieve for visual evaluation of the remaining seeds with the aid of tweezers.

Speed of germination index - performed by means of daily counts during the germination test, according to formula described by Maguire (1962). The initial, final and mean times and the germination synchrony according to formulas of Santana and Ranal were also obtained (2004).
In the statistical procedure, temperatures of $15,20,25,20-30$ and $15-25{ }^{\circ} \mathrm{C}$ were studied in independent experiments, using a randomized complete block design with four replicates. In all experiments, four lots and two substrates distributed in the factorial scheme ( $4 \times 2)$ were evaluated. After obtaining data, analyses of variance of each experiment were performed.

After individual analyses, combined analyses were programmed to evaluate the effect of temperature on germination. The combined analysis of experiments was carried out, as it was not possible to randomize the temperatures in different germination chambers, in addition, due to the importance of temperature on the germination process, both regarding total germination and the speed of germination, in order to identify the best temperature for the germination of forage pea seeds. For comparison of means, the Tukey test was used at 5 and $1 \%$ probability.

\section{RESULTS AND DISCUSSION}

The summary of the analysis of variance of the normal and abnormal seedlings data, dead and dormant seeds, initial, final and average time, germination speed index (SGI) and synchrony obtained in the conjoint analysis of the experiments conducted with different temperatures are presented in Table 1. It was noted by the results that there was significant interaction by the $\mathrm{F}$ test, between substrates and temperatures for dead seeds and final and average times; between lots and temperatures for abnormal seedlings, dead seeds and average time. Substrates, lots and temperatures for normal seedlings, initial time, SGI and synchrony, also presented interaction. 
Table 1. Summary of the analysis of variance of the data percentage of normal and abnormal seedlings, dormant and dead seeds was counted, initial, final and mean times, Speed of germination index (SGI) and the germination synchrony (S), obtained in the conjoint analysis of the experiments conducted with different temperatures.

\begin{tabular}{|c|c|c|c|c|c|c|c|c|c|c|}
\hline \multirow{3}{*}{ SV } & \multirow{3}{*}{ DF } & \multicolumn{9}{|c|}{ Mean square } \\
\hline & & \multicolumn{2}{|l|}{ Seedlings } & \multicolumn{2}{|l|}{ Seeds } & \multicolumn{3}{|l|}{ Time } & \multirow{2}{*}{ SGI } & \multirow{2}{*}{ GS } \\
\hline & & Normal & Abnormal & Dead & Dormant & Initial & Final & Mean & & \\
\hline Substrate (S) & 1 & $135,1^{* *}$ & $5,3^{\text {ns }}$ & $1709,6^{* *}$ & $752,6^{* *}$ & $0,1^{\mathrm{ns}}$ & $42,0^{* * *}$ & ${ }^{*} 1,6^{* *}$ & $55,3^{* *}$ & $0,0^{\mathrm{ns}}$ \\
\hline Lot $(\mathrm{L})$ & 3 & $33540,0^{* * *}$ & $603,8^{* * *}$ & $12399,9^{* *}$ & $2785,3^{* *}$ & $10,3^{* *}$ & $12,2^{* *}$ & * $16,8^{* *}$ & $2015,7^{* *}$ & $0,2^{* *}$ \\
\hline$S \times L$ & 3 & $23,1^{\mathrm{ns}}$ & $41,1^{\mathrm{ns}}$ & $378,2^{* *}$ & $361,7^{* *}$ & $0,6^{* *}$ & $0,3^{\mathrm{ns}}$ & $0,3^{*}$ & $13,5^{* *}$ & $0,1^{* *}$ \\
\hline \multicolumn{2}{|c|}{ Temperature (T) 4} & $360,6^{* *}$ & $59,4^{*}$ & $711,2^{* *}$ & $13,6^{\mathrm{ns}}$ & $24,2^{* *}$ & $50,1^{* *}$ & * $39,8^{* *}$ & $198,1^{* *}$ & $0,1^{* *}$ \\
\hline $\mathrm{S} \times \mathrm{T}$ & 4 & $166,7^{* *}$ & $10,4^{\mathrm{ns}}$ & $184,5^{* *}$ & $25,4^{\mathrm{ns}}$ & $3,2^{* *}$ & $4,2^{*}$ & $1,8^{* *}$ & $5,0^{* *}$ & $0,1^{* *}$ \\
\hline $\mathrm{L} \times \mathrm{T}$ & 12 & $56,7^{* *}$ & $64,3^{* *}$ & $205,0^{* *}$ & $21,3^{\mathrm{ns}}$ & $0,7^{* *}$ & $2,3^{\mathrm{ns}}$ & $1,3^{* *}$ & $19,1^{* *}$ & $0,1^{* *}$ \\
\hline$S \times L \times T$ & 12 & $68,2^{* *}$ & $9,2^{\mathrm{ns}}$ & $55,6^{\mathrm{ns}}$ & $19,3^{\mathrm{ns}}$ & $0,2^{*}$ & $0,6^{\mathrm{ns}}$ & $0,2^{\mathrm{ns}}$ & $6,0^{* *}$ & $0,1^{*}$ \\
\hline Block (T) & 15 & $23,4^{\mathrm{ns}}$ & $7,9^{\mathrm{ns}}$ & $70,0^{*}$ & $42,2 *$ & $0,1^{\mathrm{ns}}$ & $1,5^{\mathrm{ns}}$ & $0,3^{* *}$ & $0,7^{\mathrm{ns}}$ & $0,0^{\mathrm{ns}}$ \\
\hline Residue & 105 & 16,1 & 17,1 & 36,8 & 19,6 & 0,1 & 1,3 & 0,1 & 0,9 & 0,0 \\
\hline CV (\%) & & 6,1 & 43,1 & 37,5 & 53,4 & 7,6 & 15,4 & 6,2 & 6,9 & 24,5 \\
\hline
\end{tabular}

**, *and ns: significant at 1\%, 5\% and non-significant according the F test, respectively. SV: Source of variation. DF: Degrees of freedom.

The seeds of the four lots of forage pea presented water contents between 9.6 and $9.9 \%$, during the installation of experiments. Thus, lots had maximum variation of $0.4 \%$ in the water content; this value is acceptable according to the tolerance table established by the Rules for Seed Testing (BRASIL, 2009). Silva et al. (2017) working with forage legume, crotalária (Crotalaria juncea $\mathrm{L}$.), reported that the small variation between water contents provides more accurate determination of the methodology and the differences between lots can be attributed to the physiological characteristics of seeds.

Table 2. Interaction between substrate and lots for variables obtained in the germination test of Pisum sativum subsp. arvense seeds conducted at temperatures of $15,20,25,20-30$ and $15-35^{\circ} \mathrm{C}$.

\begin{tabular}{|c|c|c|c|c|c|}
\hline \multirow{2}{*}{ Variables } & \multirow{2}{*}{ Substrates } & \multicolumn{4}{|l|}{ Lots } \\
\hline & & 1 & 2 & 3 & 4 \\
\hline \multicolumn{6}{|l|}{$15^{\circ} \mathrm{C}$} \\
\hline \multirow{2}{*}{ Germination (\%) } & Paper & $98 \mathrm{aA}^{1}$ & $85 \mathrm{aB}$ & $64 \mathrm{aC}$ & $33 \mathrm{aD}$ \\
\hline & Sand & $95 \mathrm{aA}$ & $88 \mathrm{aB}$ & $57 \mathrm{bC}$ & $38 \mathrm{aD}$ \\
\hline \multirow{2}{*}{ Dead seed $(\%)$} & Paper & $0 \mathrm{aA}$ & $1 \mathrm{aA}$ & $8 \mathrm{aA}$ & $18 \mathrm{aB}$ \\
\hline & Sand & $0 \mathrm{aA}$ & $2 \mathrm{aA}$ & $19 \mathrm{bB}$ & $30 \mathrm{bC}$ \\
\hline \multirow{2}{*}{ Speed of germination index } & Paper & $16,75 \mathrm{aA}$ & $14,30 \mathrm{aB}$ & $8,32 \mathrm{aC}$ & $3,71 \mathrm{aD}$ \\
\hline & Sand & $15,34 \mathrm{bA}$ & $14,70 \mathrm{aA}$ & $8,37 \mathrm{aB}$ & $4,57 \mathrm{aC}$ \\
\hline \multirow{2}{*}{ Synchrony } & Paper & $0,39 \mathrm{bAB}$ & $0,40 \mathrm{aA}$ & $0,24 \mathrm{bC}$ & $0,29 \mathrm{aBC}$ \\
\hline & Sand & $0,52 \mathrm{aA}$ & $0,39 \mathrm{aB}$ & $0,34 \mathrm{aB}$ & $0,22 \mathrm{aC}$ \\
\hline \multicolumn{6}{|l|}{$20^{\circ} \mathrm{C}$} \\
\hline \multirow{2}{*}{ Germination (\%) } & Paper & $96 \mathrm{aA}$ & $86 \mathrm{aB}$ & $63 \mathrm{aC}$ & $30 \mathrm{bD}$ \\
\hline & Sand & $94 \mathrm{aA}$ & $84 \mathrm{aB}$ & $60 \mathrm{aC}$ & $38 \mathrm{aD}$ \\
\hline \multirow{2}{*}{ Initial time (days) } & Paper & $3,0 \mathrm{bB}$ & $3,0 \mathrm{bB}$ & $4,0 \mathrm{aA}$ & $4,5 \mathrm{aA}$ \\
\hline & Sand & $4,0 \mathrm{aB}$ & $4,0 \mathrm{aB}$ & $4,0 \mathrm{aB}$ & $4,8 \mathrm{aA}$ \\
\hline Speed of germination index & Paper & $25,34 \mathrm{aA}$ & $22,60 \mathrm{aB}$ & $14,03 \mathrm{aC}$ & $5,67 \mathrm{aD}$ \\
\hline
\end{tabular}




\begin{tabular}{|c|c|c|c|c|c|}
\hline \multirow{3}{*}{ Synchrony } & Sand & $21,96 \mathrm{bA}$ & $19,60 \mathrm{bB}$ & $12,56 \mathrm{bC}$ & $6,75 \mathrm{aD}$ \\
\hline & Paper & $0,45 \mathrm{aA}$ & $0,40 \mathrm{bA}$ & $0,45 \mathrm{aA}$ & $0,41 \mathrm{aA}$ \\
\hline & Sand & $0,58 \mathrm{aA}$ & $0,59 \mathrm{aA}$ & $0,40 \mathrm{aAB}$ & $0,24 \mathrm{bB}$ \\
\hline \multicolumn{6}{|l|}{$25^{\circ} \mathrm{C}$} \\
\hline \multirow{2}{*}{ Germination (\%) } & Paper & $94 \mathrm{aA}$ & $89 \mathrm{aB}$ & $51 \mathrm{aB}$ & $24 \mathrm{aC}$ \\
\hline & Sand & $94 \mathrm{aA}$ & $82 \mathrm{aB}$ & $56 \mathrm{aC}$ & $23 \mathrm{aD}$ \\
\hline \multirow{2}{*}{ Dead seeds $(\%)$} & Paper & $2 \mathrm{aA}$ & $2 \mathrm{aB}$ & $24 \mathrm{aB}$ & $40 \mathrm{aC}$ \\
\hline & Sand & $1 \mathrm{aA}$ & $6 \mathrm{bA}$ & $34 \mathrm{bB}$ & $56 \mathrm{bC}$ \\
\hline \multirow{2}{*}{ Dormant seeds $(\%)$} & Paper & $0 \mathrm{aA}$ & $2 \mathrm{aA}$ & $19 \mathrm{bB}$ & $24 \mathrm{bB}$ \\
\hline & Sand & $0 \mathrm{aA}$ & $5 \mathrm{aAB}$ & $3 \mathrm{aA}$ & $10 \mathrm{aB}$ \\
\hline \multirow{2}{*}{ Speed of germination index } & Paper & $25,08 \mathrm{aA}$ & $24,90 \mathrm{aA}$ & $12,08 \mathrm{aB}$ & $5,23 \mathrm{aC}$ \\
\hline & Sand & $25,16 \mathrm{aA}$ & $20,90 \mathrm{bB}$ & $13,20 \mathrm{aC}$ & $4,90 \mathrm{aD}$ \\
\hline \multicolumn{6}{|l|}{$20-30{ }^{\circ} \mathrm{C}$} \\
\hline \multirow{2}{*}{ Germination (\%) } & Paper & $94 \mathrm{aA}$ & $81 \mathrm{aB}$ & $45 \mathrm{bC}$ & $23 \mathrm{aD}$ \\
\hline & Sand & $93 \mathrm{aA}$ & $75 \mathrm{aB}$ & $54 \mathrm{aC}$ & $28 \mathrm{aD}$ \\
\hline \multirow{2}{*}{ Speed of germination index } & Paper & $21,50 \mathrm{aA}$ & $18,97 \mathrm{aB}$ & $9,71 \mathrm{aC}$ & $4,65 \mathrm{aD}$ \\
\hline & Sand & $17,40 \mathrm{bA}$ & $14,28 \mathrm{bB}$ & $10,33 \mathrm{aC}$ & $4,84 \mathrm{aD}$ \\
\hline \multirow{2}{*}{ Synchrony } & Paper & $0,45 \mathrm{aA}$ & $0,43 \mathrm{aA}$ & $0,55 \mathrm{aA}$ & $0,64 \mathrm{aA}$ \\
\hline & Sand & $0,54 \mathrm{aA}$ & $0,52 \mathrm{aA}$ & $0,48 \mathrm{aA}$ & $0,35 \mathrm{bA}$ \\
\hline \multicolumn{6}{|l|}{$15-25^{\circ} \mathrm{C}$} \\
\hline \multirow{2}{*}{ Dead seeds $(\%)$} & Paper & $0 \mathrm{aA}$ & $1 \mathrm{aA}$ & $11 \mathrm{aA}$ & $26 \mathrm{aB}$ \\
\hline & Sand & $3 \mathrm{aA}$ & $5 \mathrm{aA}$ & $33 \mathrm{bB}$ & $53 \mathrm{bC}$ \\
\hline \multirow{2}{*}{ Dormant seeds (\%) } & Paper & $1 \mathrm{aA}$ & $2 \mathrm{aA}$ & $20 \mathrm{aB}$ & $22 \mathrm{bB}$ \\
\hline & Sand & $2 \mathrm{aA}$ & $2 \mathrm{aA}$ & $7 \mathrm{aAB}$ & $14 \mathrm{aB}$ \\
\hline
\end{tabular}

${ }^{1}$ Means followed by the same letter, lowercase in the column and uppercase in the row, for each temperature, do not differ by the Tukey test at $5 \%$ probability.

Table 2 shows the interaction between substrate and lots. For temperature of $15{ }^{\circ} \mathrm{C}$, these factors had interaction for germination, dead seed, speed of germination index and synchrony. The germination percentage had a decrease from lot 1 to 4 , with difference between substrates only in lot 3, for which paper showed better result. This germination behavior is similar in other temperatures, except that difference between substrates was verified only at $20-30{ }^{\circ} \mathrm{C}$, with seeds sown in sand with higher germination and no interaction between factors for this variable at temperature of $15-25^{\circ} \mathrm{C}$ was verified.

Higher percentages of dead seeds were observed at temperatures of 15,25 and $15-25{ }^{\circ} \mathrm{C}$ in sand substrate in lots 3 and 4 . Similar result was verified for percentage of dead seeds at temperatures of 25 and $15-25{ }^{\circ} \mathrm{C}$ in paper substrate for lots 3 and 4.

The paper roll substrate provided seeds of lot 1 higher speeds of germination at all temperatures when compared to the other lots and to the sand substrate, except at $25{ }^{\circ} \mathrm{C}$, in which it did not differ between substrates used and in this temperature, lots 1 and 2 provided similar speed of germination on the paper substrate. It is also noteworthy that at temperature of $15-25{ }^{\circ} \mathrm{C}$, there was no interaction between factors for this variable.

The germination synchrony was not efficient in the differentiation of lots and substrates at temperatures of 15,20 and $20-30{ }^{\circ} \mathrm{C}$, since there were conflicting and different results compared to the other tests, classifying lot 4 (worse quality) equal to lot 1 (best quality).

The isolated effect of substrates (Table 3) on mean and final time was lower on paper substrate at temperatures of $20,25,20-30{ }^{\circ} \mathrm{C}$, and shorter initial time was observed in seed sown on paper at $20-30{ }^{\circ} \mathrm{C}$ and in sand at $15-25{ }^{\circ} \mathrm{C}$. The paper substrate provided higher percentage and speed of germination for forage pea seeds sown at $15-25{ }^{\circ} \mathrm{C}$, for variables germination, speed of germination index and synchrony. 
Table 3. Means of variables obtained in the germination test of Pisum sativum subsp. arvense seeds conducted in two substrates at temperatures of $20,25,20-30$ and $15-35^{\circ} \mathrm{C}$.

\begin{tabular}{|c|c|c|c|c|c|c|}
\hline \multirow{2}{*}{$\frac{\text { Variables }}{20^{\circ} \mathrm{C}}$} & & \multicolumn{2}{|c|}{ Substrates } & \multirow{2}{*}{$\frac{\text { Variables }}{25^{\circ} \mathrm{C}}$} & \multicolumn{2}{|c|}{ Substrates } \\
\hline & & & & & & \\
\hline \multirow{4}{*}{ Time (days) } & & Paper & $4,5 \mathrm{a}^{1}$ & \multirow{2}{*}{ Mean } & Paper & $4,1 \mathrm{a}$ \\
\hline & & Sand & $4,9 \mathrm{~b}$ & & Sand & $4,3 \mathrm{~b}$ \\
\hline & \multirow{2}{*}{ Final } & Paper & $5,9 \mathrm{a}$ & lime (days) - - - & Paper & $5,8 \mathrm{a}$ \\
\hline & & Sand & $7,0 \mathrm{~b}$ & & Sand & $6,9 \mathrm{~b}$ \\
\hline \multicolumn{4}{|l|}{$20-30{ }^{\circ} \mathrm{C}$} & \multicolumn{3}{|l|}{$15-25^{\circ} \mathrm{C}$} \\
\hline \multirow{2}{*}{\multicolumn{2}{|c|}{ Dormant seeds (\%) }} & Paper & $10,0 \mathrm{~b}$ & \multirow{2}{*}{ Germination (\%) } & Paper & $70 \mathrm{a}$ \\
\hline & & Sand & $6,0 \mathrm{a}$ & & Sand & $61 \mathrm{~b}$ \\
\hline \multirow{2}{*}{\multicolumn{2}{|c|}{ Initial }} & Paper & $3,6 \mathrm{a}$ & \multirow{2}{*}{ Initial time (days) } & Paper & $5,0 \mathrm{~b}$ \\
\hline & & Sand & $4,3 \mathrm{~b}$ & & Sand & $4,3 \mathrm{a}$ \\
\hline \multirow{4}{*}{ Time (days) } & & Paper & $4,6 \mathrm{a}$ & \multirow{2}{*}{\multicolumn{2}{|c|}{$\begin{array}{l}\text { Speed of germinationPaper } \\
\text { index }\end{array}$}} & $12,90 \mathrm{a}$ \\
\hline & Mean & Sand & $5,5 \mathrm{~b}$ & & & $11,60 \mathrm{~b}$ \\
\hline & & Paper & $6,0 \mathrm{a}$ & \multirow{2}{*}{ Synchrony } & Paper & $0,5 \bar{a}$ \\
\hline & Final & Sand & $8,1 \mathrm{~b}$ & & Sand & $0,43 \mathrm{~b}$ \\
\hline
\end{tabular}

${ }^{1}$ Means followed by the same letter, lowercase in the column, for each temperature, do not differ by the Tukey test at $5 \%$ probability.

The best germination performance of forage pea seeds in paper substrate can be attributed to the vegetative structure's development lower physical impairment and higher aeration of this substrate in comparison to sand. Probably for this species, these factors must be more important than the contact surface, which favors water absorption (PACHECO et al., 2014; OLIVEIRA et al., 2016). In the germination of forage pea, the plumule is folded and fixed to the epicotyl, which grows by perforating the sand layer, dragging the primary leaves (BRASIL, 2009; CARVALHO; NAKAGAWA, 2012). Thus, the seedling needs more energy to break the sand layer during the germination process than in paper subtract, a fact that may explain the lower percentage of dormant seeds verified for seeds sown in sand at temperature of $20-30{ }^{\circ} \mathrm{C}$.

When variables that were influenced only by the lot factor were evaluated, Table 4 , it was verified that lot 1 and 2 are of high quality, lot 3 of intermediate quality and lot 4 of low quality. Since there is a decrease in speed and percentage of germination from lot 1 to 4 , the opposite performance was verified for variables abnormal seedlings, dead and dormant seeds.

Table 4. Means of variables obtained in the germination test of four Pisum sativum subsp. arvense seed lots conducted at temperatures of $15,20,25,20-30$ and $15-35^{\circ} \mathrm{C}$.

\begin{tabular}{|c|c|c|c|c|c|}
\hline \multirow{2}{*}{ Variables } & & \multicolumn{4}{|l|}{ Lots } \\
\hline & & 1 & 2 & 3 & 4 \\
\hline \multicolumn{6}{|l|}{$15^{\circ} \mathrm{C}$} \\
\hline \multicolumn{2}{|c|}{ Abnormal seedlings (\%) } & $4 \mathrm{~A}^{1}$ & $11 \mathrm{~B}$ & $13 \mathrm{~B}$ & $18 \mathrm{C}$ \\
\hline \multicolumn{2}{|c|}{ Dormant seeds $(\%)$} & $0 \mathrm{~A}$ & $1 \mathrm{~A}$ & $13 \mathrm{~B}$ & $23 \mathrm{C}$ \\
\hline \multirow{3}{*}{ Time (days) } & Initial & $5,1 \mathrm{~A}$ & $5,0 \mathrm{~A}$ & $5,8 \mathrm{~B}$ & $6,9 \mathrm{C}$ \\
\hline & Mean & $6,1 \mathrm{~A}$ & $6,1 \mathrm{~A}$ & $7,4 \mathrm{~B}$ & $8,8 \mathrm{C}$ \\
\hline & Final & $8,1 \mathrm{~A}$ & $8,6 \mathrm{~A}$ & $9,8 \mathrm{AB}$ & $11,1 \mathrm{C}$ \\
\hline \multicolumn{6}{|l|}{$20^{\circ} \mathrm{C}$} \\
\hline \multicolumn{2}{|c|}{ Abnormal seedlings (\%) } & $5 \mathrm{~A}$ & $10 \mathrm{~A}$ & $8 \mathrm{~A}$ & $18 \mathrm{~B}$ \\
\hline \multicolumn{2}{|c|}{ Dead seeds $(\%)$} & $0 \mathrm{~A}$ & $3 \mathrm{~A}$ & $21 \mathrm{~B}$ & $30 \mathrm{~B}$ \\
\hline
\end{tabular}




\begin{tabular}{|c|c|c|c|c|c|}
\hline \multicolumn{2}{|c|}{ Dormant seeds $(\%)$} & $0 \mathrm{~A}$ & $3 \mathrm{~A}$ & $10 \mathrm{~B}$ & $18 \mathrm{C}$ \\
\hline \multirow{2}{*}{ Time (days) } & Mean & $4,1 \mathrm{~A}$ & $4,1 \mathrm{~A}$ & $4,8 \mathrm{~B}$ & $5,6 \mathrm{C}$ \\
\hline & Final & $5,9 \mathrm{~A}$ & $6,0 \mathrm{AB}$ & $6,6 \mathrm{AB}$ & $7,3 \mathrm{~B}$ \\
\hline \multicolumn{6}{|l|}{$25^{\circ} \mathrm{C}$} \\
\hline \multicolumn{2}{|c|}{ Abnormal seedlings (\%) } & $5 \mathrm{~A}$ & $7 \mathrm{AB}$ & $7 \mathrm{AB}$ & $12 \mathrm{~B}$ \\
\hline \multirow{2}{*}{ Time (days) } & Initial & $3,0 \mathrm{~A}$ & $3,0 \mathrm{~A}$ & $3,8 \mathrm{~B}$ & $4,1 \mathrm{C}$ \\
\hline & Mean & $3,9 \mathrm{~A}$ & $3,9 \mathrm{~A}$ & $4,3 \mathrm{~B}$ & $4,8 \mathrm{C}$ \\
\hline \multicolumn{2}{|l|}{ Synchrony } & $0,46 \mathrm{AB}$ & $0,52 \mathrm{AB}$ & $0,60 \mathrm{~A}$ & $0,42 \mathrm{~B}$ \\
\hline \multicolumn{6}{|l|}{$20-30^{\circ} \mathrm{C}$} \\
\hline \multicolumn{2}{|c|}{ Abnormal seedlings (\%) } & $5 \mathrm{~A}$ & $14 \mathrm{C}$ & $10 \mathrm{AB}$ & $8 \mathrm{AB}$ \\
\hline \multicolumn{2}{|c|}{ Dead seeds $(\%)$} & $1 \mathrm{~A}$ & $4 \mathrm{~A}$ & $29 \mathrm{~B}$ & $49 \mathrm{C}$ \\
\hline \multicolumn{2}{|c|}{ Dormant seeds (\%) } & $1 \mathrm{~A}$ & $4 \mathrm{AB}$ & $11 \mathrm{BC}$ & $17 \mathrm{C}$ \\
\hline \multirow{2}{*}{ Time (days) } & Initial & $3,5 \mathrm{~A}$ & $3,5 \mathrm{~A}$ & $4,4 \mathrm{~B}$ & $4,4 \mathrm{~B}$ \\
\hline & Mean & $5,0 \mathrm{AB}$ & $4,9 \mathrm{~A}$ & $5,0 \mathrm{AB}$ & $5,4 \mathrm{~B}$ \\
\hline \multicolumn{6}{|l|}{$15-25^{\circ} \mathrm{C}$} \\
\hline \multicolumn{2}{|c|}{$\overline{\text { Germination }(\%)}$} & $93 \mathrm{~A}$ & $83 \mathrm{~B}$ & $55 \mathrm{C}$ & $31 \mathrm{D}$ \\
\hline \multicolumn{2}{|c|}{ Abnormal seedlings $(\%)$} & $4 \mathrm{~A}$ & $12 \mathrm{~B}$ & $10 \mathrm{AB}$ & $12 \mathrm{~B}$ \\
\hline \multicolumn{2}{|c|}{ Mean time(days) } & $5,2 \mathrm{~A}$ & $5,2 \mathrm{~A}$ & $5,8 \mathrm{~B}$ & $6,4 \mathrm{C}$ \\
\hline \multicolumn{2}{|c|}{ Speed of germination index } & $18,30 \mathrm{~A}$ & $16,20 \mathrm{~B}$ & $9,69 \mathrm{C}$ & $4,88 \mathrm{D}$ \\
\hline \multicolumn{2}{|l|}{ Synchrony } & $0,66 \mathrm{~A}$ & $0,65 \mathrm{~A}$ & $0,35 \mathrm{~B}$ & $0,33 \mathrm{~B}$ \\
\hline
\end{tabular}

${ }^{1}$ Means followed by the same letter, uppercase in the row, for each temperature, do not differ by the Tukey test at $5 \%$ probability.

The identification of high quality lots is a prime factor during seed production programs as an important strategy for seed quality control as they help ensuring of the plant population in the field (SILVA et al., 2017).

Aiming at identifying the best temperature for the germination of forage pea seeds, the combined analyses of experiments was performed, because it is not possible to randomize the temperatures in different germination chambers. The effect of temperature on interaction with the substrate and seed quality (Lot) on germination, initial time, speed of germination index and germination synchrony was verified in combined analyses (Table 5).

Table 5. Combined analyses of the interaction between temperature (T), substrates and lots in the evaluation of variables of the germination test of Pisum sativum subsp. arvense seeds.

\begin{tabular}{|c|c|c|c|c|c|c|c|c|c|}
\hline \multirow{3}{*}{ Variables } & \multirow{3}{*}{$\mathrm{T}^{\circ} \mathrm{C}$} & \multicolumn{8}{|c|}{ Lots/Substrates } \\
\hline & & \multicolumn{2}{|c|}{1} & \multicolumn{2}{|l|}{2} & \multicolumn{2}{|l|}{3} & \multicolumn{2}{|l|}{4} \\
\hline & & Paper & Sand & Paper & Sand & Paper & Sand & Paper & Sand \\
\hline \multirow{6}{*}{ 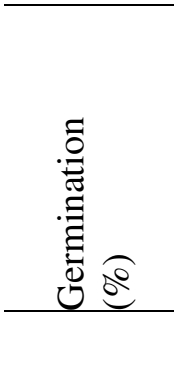 } & 15 & $98 \mathrm{a}^{1}$ & $95 \mathrm{a}$ & $85 \mathrm{ab}$ & $88 \mathrm{a}$ & $64 \mathrm{a}$ & $57 \mathrm{a}$ & $33 \mathrm{ab}$ & $38 \mathrm{a}$ \\
\hline & 20 & $96 \mathrm{a}$ & $94 \mathrm{a}$ & $86 \mathrm{ab}$ & $84 \mathrm{a}$ & $63 a$ & $60 \mathrm{a}$ & $30 \mathrm{bc}$ & $38 \mathrm{a}$ \\
\hline & 25 & $94 \mathrm{a}$ & $94 \mathrm{a}$ & $89 \mathrm{a}$ & $83 \mathrm{ab}$ & $51 \mathrm{~b}$ & $56 \mathrm{a}$ & $24 \mathrm{c}$ & $23 \mathrm{~b}$ \\
\hline & $20-30$ & $94 \mathrm{a}$ & $93 \mathrm{a}$ & $81 \mathrm{~b}$ & $75 \mathrm{~b}$ & $45 \mathrm{~b}$ & $54 \mathrm{ab}$ & $23 \mathrm{c}$ & $28 \mathrm{~b}$ \\
\hline & $15-25$ & $96 \mathrm{a}$ & $91 \mathrm{a}$ & $85 a b$ & $81 \mathrm{ab}$ & $62 \mathrm{a}$ & $48 \mathrm{~b}$ & $39 \mathrm{a}$ & $24 \mathrm{~b}$ \\
\hline & 15 & $5,0 \mathrm{~b}$ & $5,3 \mathrm{c}$ & $5,0 \mathrm{~b}$ & $5,0 \mathrm{c}$ & $6,0 \mathrm{c}$ & $5,5 \mathrm{~d}$ & $7,3 \mathrm{c}$ & $6,5 \mathrm{~b}$ \\
\hline \multirow{2}{*}{ : 急导 疍 } & 20 & $3,0 \mathrm{a}$ & $4,0 \mathrm{~b}$ & $3,0 \mathrm{a}$ & $4,0 \mathrm{~b}$ & $4,0 \mathrm{a}$ & $4,0 \mathrm{ab}$ & $4,5 \mathrm{a}$ & $4,6 \mathrm{a}$ \\
\hline & 25 & $3,0 \mathrm{a}$ & $3,0 \mathrm{a}$ & $3,0 \mathrm{a}$ & $3,0 \mathrm{a}$ & $4,0 \mathrm{a}$ & $3,5 \mathrm{a}$ & $4,0 \mathrm{a}$ & $4,3 \mathrm{a}$ \\
\hline
\end{tabular}




\begin{tabular}{|c|c|c|c|c|c|c|c|c|c|}
\hline & $20-30$ & $3,0 \mathrm{a}$ & $4,0 \mathrm{~b}$ & $3,0 \mathrm{a}$ & $4,0 \mathrm{~b}$ & $4,0 \mathrm{a}$ & $4,8 \mathrm{c}$ & $4,3 \mathrm{a}$ & $4,5 \mathrm{a}$ \\
\hline & $15-25$ & $5,0 \mathrm{~b}$ & $4,0 \mathrm{~b}$ & $5,0 \mathrm{~b}$ & $4,3 \mathrm{~b}$ & $5,0 \mathrm{~b}$ & $4,3 \mathrm{bc}$ & $5,3 \mathrm{~b}$ & $4,5 \mathrm{a}$ \\
\hline \multirow{5}{*}{ 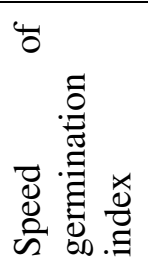 } & 15 & $16,8 \mathrm{c}$ & $15,3 \mathrm{~d}$ & $14,3 \mathrm{e}$ & $14,7 \mathrm{~b}$ & $8,3 \mathrm{~d}$ & $8,4 \mathrm{c}$ & $3,7 \mathrm{~b}$ & $4,6 \mathrm{~b}$ \\
\hline & 20 & $25,4 \mathrm{a}$ & $21,9 \mathrm{~b}$ & $22,6 \mathrm{~b}$ & $19,6 \mathrm{a}$ & $14,0 \mathrm{a}$ & $12,6 \mathrm{a}$ & $5,7 \mathrm{a}$ & $6,6 \mathrm{a}$ \\
\hline & 25 & $25,1 \mathrm{a}$ & $25,2 \mathrm{a}$ & $24,9 \mathrm{a}$ & $20,8 \mathrm{a}$ & $12,1 \mathrm{~b}$ & $13,2 \mathrm{a}$ & $5,2 \mathrm{ab}$ & $4,9 a b$ \\
\hline & $20-30$ & $21,5 \mathrm{~b}$ & $17,4 \mathrm{c}$ & $18,9 \mathrm{c}$ & $14,3 \mathrm{~b}$ & $9,7 \mathrm{~cd}$ & $10,3 \mathrm{~b}$ & $4,7 \mathrm{ab}$ & $4,8 \mathrm{~b}$ \\
\hline & $15-25$ & $18,6 \mathrm{c}$ & $17,9 \mathrm{c}$ & $16,6 \mathrm{~d}$ & $15,8 \mathrm{~b}$ & $10,8 \mathrm{bc}$ & $8,6 \mathrm{bc}$ & $5,8 \mathrm{a}$ & $3,9 \mathrm{~b}$ \\
\hline \multirow{5}{*}{ 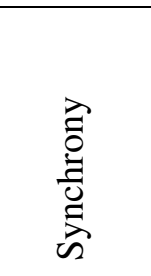 } & 15 & $0,39 \mathrm{~b}$ & $0,52 \mathrm{a}$ & $0,40 \mathrm{~b}$ & $0,39 \mathrm{a}$ & $0,24 \mathrm{c}$ & $0,34 \mathrm{~b}$ & $0,29 \mathrm{~b}$ & $0,22 \mathrm{a}$ \\
\hline & 20 & $0,45 \mathrm{~b}$ & $0,58 \mathrm{a}$ & $0,40 \mathrm{~b}$ & $0,59 \mathrm{a}$ & $0,45 \mathrm{abc}$ & $0,40 a b$ & $0,41 \mathrm{ab}$ & $0,24 \mathrm{a}$ \\
\hline & 25 & $0,37 \mathrm{~b}$ & $0,54 \mathrm{a}$ & $0,42 \mathrm{~b}$ & $0,61 \mathrm{a}$ & $0,60 \mathrm{a}$ & $0,60 \mathrm{a}$ & $0,44 \mathrm{ab}$ & $0,39 \mathrm{a}$ \\
\hline & $20-30$ & $0,45 \mathrm{~b}$ & $0,54 \mathrm{a}$ & $0,43 \mathrm{~b}$ & $0,52 \mathrm{a}$ & $0,55 \mathrm{a}$ & $0,48 \mathrm{ab}$ & $0,64 \mathrm{a}$ & $0,35 \mathrm{a}$ \\
\hline & $15-25$ & $0,75 \mathrm{a}$ & $0,57 \mathrm{a}$ & $0,79 \mathrm{a}$ & $0,50 \mathrm{a}$ & $0,36 \mathrm{~b}$ & $0,34 \mathrm{~b}$ & $0,36 \mathrm{~b}$ & $0,30 \mathrm{a}$ \\
\hline
\end{tabular}

${ }^{1}$ Means followed by the same letter, lowercase in the column, for each substrate, do not differ by the Tukey test at 5\% probability.

Temperature had a significant effect on germination as the quality of lots reduced, with better performance of seeds under conditions of mild temperatures (15 and $20{ }^{\circ} \mathrm{C}$ ). This result is probably because pea is originated in the Middle East, so that the culture develops and produces well in regions with temperature between 4 and $30{ }^{\circ} \mathrm{C}$ and temperature of $18{ }^{\circ} \mathrm{C}$ is considered ideal (VIEIRA et al., 2007; CARVALHO et al., 2012; TORALES et al., 2014).

In relation to the speed of germination, it was observed for the initial time and speed of germination index that the temperature of $15^{\circ} \mathrm{C}$ for all lots in both substrates was the one that presented worse performance with the longest time to start germination and lower speed of germination index. For these variables, the best performance of constant temperatures of 20 and $25^{\circ} \mathrm{C}$ is highlighted, and for the initial time, temperature of $20-30{ }^{\circ} \mathrm{C}$ is also highlighted.

The best seed performance at $25{ }^{\circ} \mathrm{C}$ for variables that evaluate speed of germination can be attributed to the fact that at higher temperatures, the water absorption and chemical reactions rates are higher, and seeds germinate more rapidly (CARVALHO; NAKAGAWA, 2012).

Therefore, the temperatures most favorable for the speed of germination were higher than those observed to obtain the maximum percentage of germination. This phenomenon can be explained by the acceleration of metabolic reactions of seeds due to the high temperatures, associated with protein denaturation and alteration of cell membranes, so that the number of seeds that can complete the germination reduces rapidly (KAPOOR et al., 2011).
The germination synchrony was influenced by temperatures mainly for paper substrate, because for sand substrate in all lots except for lot 3, temperature did not exert significant influence. On the paper substrate for lots 1 and 2, the germination of seeds was more synchronized at temperature of $15-25{ }^{\circ} \mathrm{C}$, that is, the germination distribution in time was more regular than in the other treatments.

Table 6 shows the effect of temperature in interaction with the substrate, on the incidence of dead seeds and final and medium germination times. For the percentage of dead seeds, it was observed that in the paper substrate, the temperature of $15^{\circ} \mathrm{C}$ obtained the lowest percentage of dead seeds, not differing only from those sown at $15-25{ }^{\circ} \mathrm{C}$. In the sand substrate, the temperatures of 15 and $20{ }^{\circ} \mathrm{C}$ presented smaller percentages of dead seeds in comparison to the other evaluated temperatures, which did not differ from each other. The availability of oxygen may be related to the higher percentages of dead seeds (ZUCARELI et al., 2009), so at higher temperatures, oxygen availability is reduced by increasing the percentage of dead seeds (GAZOLA et al., 2014). 
Table 6. Combined analysis of the interaction between temperature $(\mathrm{T})$ and substrates, as well as temperature and lots in the evaluation of variables of the germination test of Pisum sativum subsp. arvense seeds

\begin{tabular}{|c|c|c|c|c|c|c|c|c|c|}
\hline \multirow{2}{*}{ Variables } & \multirow{2}{*}{$\mathrm{T}\left({ }^{\circ} \mathrm{C}\right)$} & \multicolumn{2}{|c|}{ Substrates } & \multirow{2}{*}{ Variables } & \multirow{2}{*}{$\mathrm{T}\left({ }^{\circ} \mathrm{C}\right)$} & \multicolumn{4}{|l|}{ Lots } \\
\hline & & Paper & Sand & & & 1 & 2 & 3 & 4 \\
\hline \multirow{5}{*}{ 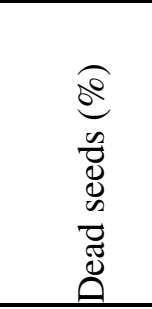 } & 15 & $7 a^{1}$ & $13 \mathrm{a}$ & \multirow{5}{*}{ 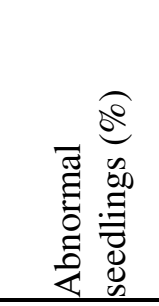 } & 15 & $4 a$ & $11 \mathrm{ab}$ & $13 \mathrm{~b}$ & $18 \mathrm{~b}$ \\
\hline & 20 & $13 b c$ & $15 \mathrm{a}$ & & 20 & $5 \mathrm{a}$ & $10 \mathrm{ab}$ & $7 \mathrm{ab}$ & $18 \mathrm{~b}$ \\
\hline & 25 & $17 \mathrm{~cd}$ & $24 b$ & & 25 & $5 a$ & $7 \mathrm{a}$ & $6 a$ & $12 \mathrm{a}$ \\
\hline & $20-30$ & $19 \mathrm{~d}$ & $22 b$ & & $20-30$ & $5 a$ & $14 \mathrm{~b}$ & $10 a b$ & $8 \mathrm{a}$ \\
\hline & $15-25$ & $9 a b$ & $23 \mathrm{~b}$ & & $15-25$ & $4 \mathrm{a}$ & $12 a b$ & $10 a b$ & $12 \mathrm{~b}$ \\
\hline \multirow{5}{*}{ 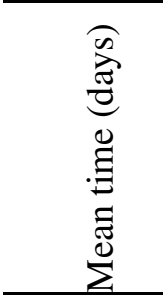 } & 15 & $7,2 \mathrm{~d}$ & $7,0 \mathrm{~d}$ & \multirow{5}{*}{ 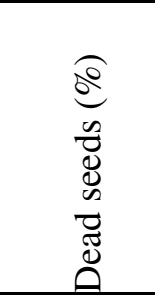 } & 15 & $0 \mathrm{a}$ & $2 a$ & $13 \mathrm{a}$ & $24 \mathrm{a}$ \\
\hline & 20 & $4,5 \mathrm{~b}$ & $4,9 \mathrm{~b}$ & & 20 & $0 \mathrm{a}$ & $3 a$ & $21 a b$ & $30 \mathrm{a}$ \\
\hline & 25 & $4,1 \mathrm{a}$ & $4,3 \mathrm{a}$ & & 25 & $1 \mathrm{a}$ & $4 \mathrm{a}$ & $29 \mathrm{~b}$ & $49 \mathrm{c}$ \\
\hline & $20-30$ & $4,6 \mathrm{~b}$ & $5,5 \mathrm{c}$ & & $20-30$ & $1 \mathrm{a}$ & $4 \mathrm{a}$ & $29 \mathrm{~b}$ & $49 \mathrm{c}$ \\
\hline & $15-25$ & $5,7 \mathrm{c}$ & $5,6 \mathrm{c}$ & & $15-25$ & $1 \mathrm{a}$ & $3 \mathrm{a}$ & $22 \mathrm{~b}$ & $39 \mathrm{~b}$ \\
\hline \multirow{5}{*}{ 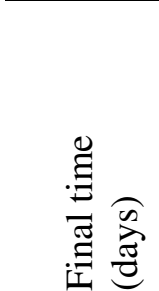 } & 15 & $9,2 \mathrm{c}$ & $9,6 \mathrm{c}$ & \multirow{5}{*}{ 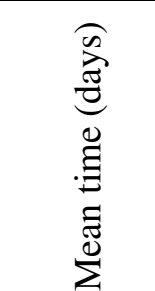 } & 15 & $6,1 \mathrm{c}$ & $6,1 \mathrm{c}$ & $7,4 \mathrm{~d}$ & $8,8 \mathrm{~d}$ \\
\hline & 20 & $5,9 \mathrm{a}$ & $7,0 \mathrm{a}$ & & 20 & $4,1 \mathrm{a}$ & $4,2 \mathrm{a}$ & $4,8 \mathrm{ab}$ & $5,6 \mathrm{~b}$ \\
\hline & 25 & $5,8 \mathrm{a}$ & $6,9 \mathrm{a}$ & & 25 & $3,9 \mathrm{a}$ & $3,9 \mathrm{a}$ & $4,3 \mathrm{a}$ & $4,8 \mathrm{a}$ \\
\hline & $20-30$ & $6,0 \mathrm{a}$ & $8,1 \mathrm{~b}$ & & $20-30$ & $5,0 \mathrm{~b}$ & $4,9 \mathrm{~b}$ & $5,0 \mathrm{~b}$ & $5,4 \mathrm{~b}$ \\
\hline & $15-25$ & $7,6 \mathrm{~b}$ & $8,0 \mathrm{ab}$ & & $15-25$ & $5,2 \mathrm{~b}$ & $5,2 \mathrm{~b}$ & $5,8 \mathrm{c}$ & $6,4 c$ \\
\hline
\end{tabular}

${ }^{1}$ Means followed by the same letter, lowercase in the column, for each substrate or lot, do not differ by the Tukey test at 5\% probability.

Lower mean and final germination times were found for seeds maintained at $25{ }^{\circ} \mathrm{C}$, regardless of substrate, except for paper substrate that this temperature was similar to 20 and $20-30{ }^{\circ} \mathrm{C}$ and in the sand substrate, temperature was similar to 20 and $15-25^{\circ} \mathrm{C}$, at the final germination time.

Table 6 shows too the effect of temperature in interaction with seed quality (Lot), on the incidence of abnormal seedlings, dead seeds and the average time for germination. For abnormal seedlings, lot 1 did not present statistical difference among temperatures. In lots 2 and 3, temperature of $25{ }^{\circ} \mathrm{C}$ provided lower percentage of abnormal seedlings in relation to temperature of 20-30 and 15 ${ }^{\circ} \mathrm{C}$, respectively, and the others were not statistically different. For lot 4, temperatures of 25 and $20-30{ }^{\circ} \mathrm{C}$ did not differ and presented smaller percentage of abnormal seedlings in relation to the other temperatures.

The percentage of dead seeds of lots 1 and 2 were similar among evaluated temperatures. Lot 3 presented lower percentage of dead seeds at $15{ }^{\circ} \mathrm{C}$ compared to temperatures of 25, 20-30 and 15-25 ${ }^{\circ} \mathrm{C}$, which did not differ from each other and temperature of $20{ }^{\circ} \mathrm{C}$ presented intermediate mean and not statistically different from the others. For lot 4 , temperatures 15 and $20{ }^{\circ} \mathrm{C}$ did not differ from each other and presented lower percentage of dead seeds in comparison to the others. Conversely, temperatures of 25 and $20-30{ }^{\circ} \mathrm{C}$ also did not show statistical difference, being the temperatures that presented the highest percentages of dead seeds in comparison to the others.

It is noteworthy that all temperatures evaluated presented more than $24 \%$ of dead seeds for lot 4, highlighting temperatures of 25 and 20-30 ${ }^{\circ} \mathrm{C}$ with almost $50 \%$ of dead seeds, being one of the evidences of low quality of this lot.

For the average germination time of forage pea, it was verified by the means of lots 1 and 2, as well as for dead seeds, that they presented the same behavior among themselves, being that in this case, differences among temperatures were observed. At temperature of $15^{\circ} \mathrm{C}$, the highest average time was obtained and the lowest at temperatures of 20 and $25{ }^{\circ} \mathrm{C}$, the latter were not different from each other. Lots 3 and 4 presented similar behavior, also showing the highest average time at temperature of $15^{\circ} \mathrm{C}$ in relation to the others. For lot 3 , the average germination time at $20{ }^{\circ} \mathrm{C}$ did not differ statistically from temperatures of 25 and $20-30{ }^{\circ} \mathrm{C}$ with the lowest average time. As for lot 4 , the lowest mean time was observed at temperature of $25^{\circ} \mathrm{C}$.

In general, temperature of $20{ }^{\circ} \mathrm{C}$ stood out in relation to the others and paper substrate presented better performance in relation to sand 
substrate mainly in relation to the speed of germination, in addition to being a substrate easy to handle because it facilitates the counting and extraction of seedlings, disposal and storage.

We can also emphasize that the first count of forage pea germination can be done on the $4^{\text {th }}$ day after sowing, since the final germination percentage of the most vigorous lot on this day presents $50 \%+$ 1 of the total germinated seedlings and can be used as an indication of vigor, in addition to reducing the sources of contamination and facilitating the final germination reading. The final germination reading can be done on the $7^{\text {th }}$ day after sowing, as from this day, germination of seedlings is stabilized.

\section{CONCLUSIONS}

The germination test for forage pea seeds should be conducted at the constant temperature 20 ${ }^{\circ} \mathrm{C}$ in roll of paper substrate with the first count and final count on the 4 th and 7 th day, respectively

RESUMO: As sementes apresentam desempenho germinativo variável em diferentes temperaturas e substratos, que são componentes básicos do teste de germinação. Dentro deste contexto, este trabalho teve como objetivo adequar a metodologia para o teste de germinação de sementes de $P$. sativum subsp. arvense. Para tanto, foram utilizados quatro lotes de sementes, cinco temperaturas $\left(15,20,25,20-30\right.$ e $\left.15-25^{\circ} \mathrm{C}\right)$ e dois substratos (rolo de papel e entre areia). O teor de água das sementes foi determinado antes das avaliações e o efeito dos lotes, substratos e temperaturas sobre a germinação foi avaliado diariamente. Calculou-se a porcentagem de germinação, de plântulas anormais, de sementes dormentes e mortas, o índice de velocidade de germinação, os tempos inicial, final e médio e sincronia. O delineamento adotado foi o de blocos casualizados, com quatro repetições e o procedimento estatístico adotado foi análise conjunta. $\mathrm{O}$ teste de germinação deve ser conduzido na temperatura constante de $20{ }^{\circ} \mathrm{C}$, em substrato rolo de papel com primeira contagem e contagem final ao $4^{\circ}$ e $7^{\circ}$ dia, respectivamente.

PALAVRAS-CHAVE: Pisum sativum subsp. arvense. Substrato. Temperatura. Controle de qualidade. Velocidade de germinação.

\section{REFERENCES}

BRASIL. Ministério da Agricultura e da Reforma Agrária. Regras para análise de sementes. Brasília, 2009. $395 p$.

CARGNELUTTI FILHO, A.; ALVES, B. M.; BURIN, C.; KLEINPAUL, J. A.; NEU, I. M. M.; SILVEIRA, D. L.; SIMÕES, F. M.; SPANHOLI, R.; MEDEIROS, L. B. Tamanho de parcela e número de repetições em ervilha forrageira. Ciência Rural, Santa Maria, v. 45, n. 7, p. 1174-1182, 2015. http://dx.doi.org/10.1590/0103$8478 \mathrm{cr} 20141043$.

CARVAlHO, A.; REZENDE, F. C.; AQUINO, R. F.; DE FREITAS, W. A.; OLIVEIRA, E. C. Produção da ervilha cultivada em ambiente protegido sob diferentes tensões de água no solo. Revista Brasileira de Engenharia Agrícola e Ambiental, Campina Grande, v. 16, n. 1, 2012. http://dx.doi.org/10.1590/S141543662012000100006.

CARVALHO, N. M.; NAKAGAWA, J. Sementes: ciência, tecnologia e produção. 5. ed. Jaboticabal: FUNEP, 2012. 590p.

COIMBRA, R. A.; TOMAZ, C. D. A.; MARTINS, C. C.; NAKAGAWA, J. Teste de germinação com acondicionamento dos rolos de papel em sacos plásticos visando a otimização dos resultados. Revista Brasileira Sementes, Londrina, v. 29, n. 1, p. 92-97, 2007. http://dx.doi.org/10.1590/S010131222007000100013.

GAZOLA, D.; ZUCARELI, C.; CAMARGO, M. C. Comportamento germinativo de sementes de cultivares de milho sob condições de hipoxia. Científica, Jaboticabal, v. 42, n. 3, p. 224-232, 2014. 
GIORDANO L. de B.; PEREIRA, A. S. A ervilha na alimentação animal. Informe Agropecuário, v.14, n.158, p.65, 1989.

KAPOOR, N.; ARYA, A.; SIDDIQUI, M. A.; KUMAR, H.; AMIR, A. Physiological and biochemical changes during seed deterioration in aged seeds of rice (Oryza sativa L.). American Journal of Plant Physiology, Meerut, v. 6, n. 1, p. 28-35, 2011. http://dx.doi.org/10.3923/ajpp.2011.28.35.

MACHADO, C. G.; MARTINS, C. C.; SANTANA, D. G.; CRUZ, S. C. S.; OLIVEIRA, S. S. C. Adequação do teste de condutividade elétrica para sementes de Pisum sativum subsp. Arvense. Ciência Rural, Santa Maria, v. 41, n. 6, p. 988-995, 2011. http://dx.doi.org/10.1590/S0103-84782011005000062.

MAGUIRE, J. D. Speed of germination-aid in selection and evaluation for seedling emergence and vigor. Crop Science, Madison, v. 2, n. 1, p. 176-177, 1962. https://doi.org/10.2135/cropsci1962.0011183X000200020033x

PINNOW, C.; BENIN, G.; VIOLA, R.; SILVA, C. D.; GUTKOSKI, L. C.; CASSOL, L. C. Qualidade industrial do trigo em resposta à adubação verde e doses de nitrogênio. Bragantia, v. 72, n. 1, p. 20-28, 2013. https://doi.org/10.1590/S0006-87052013005000019

SANTANA, D. G.; RANAL, M. A. Análise da germinação: um enfoque estatístico. Brasília: Universidade de Brasília, 2004. 248p.

SILVA, C. B.; BARBOSA, R. M.; VIEIRA, R. D. Accelerated aging as vigor test for sunn hemp seeds. Ciência Rural, Santa Maria, v. 47, n. 1, p. 1-6, 2017. http://dx.doi.org/10.1590/0103-8478cr20151527.

TAN, M.; Koc, A.; DUMLU GÜL, Z. ELKOCA, E.; GUL, I. Determination of dry matter yield and yield components of local forage pea (Pisum sativum ssp. arvense L.) ecotypes. Journal of Agricultural Science, v. 19, n. 4, p. 289-296, 2013.

TOMAZ, C. A.; MARTINS, C. C.; SANCHES, M. F. G.; VIEIRA, R. D. Time reduction for surinam grass seed germination test. Ciência e Agrotecnologia, Lavras, v. 39, n. 5, p. 488-497, 2015.

http://dx.doi.org/10.1590/S1413-70542015000500007.

TOMAZ, C. A.; MARTINS, C. C.; SILVA, G. Z.; VIEIRA, R. D. Period of time taken by Brachiaria humidicola (Rendle) Scheweick seed to complete germination. Semina: Ciências Agrárias, Londrina, v. 37, n. 2, p. 693-700, 2016. http://dx.doi.org/10.5433/1679-0359.2016v37n2p693.

TORALES, E. P.; ZÁRATE, N. A. H.; VIEIRA, M. D. C.; GUTIERREZ, R. S.; GASSI, R. P.; TABALDI, L. A. Arranjo de plantas e número de sementes por cova na produção agroeconômica de ervilha. Semina: Ciências Agrárias, Londrina, v. 35, n. 6, p. 2955-2966, 2014. http://dx.doi.org/10.5433/16790359.2014v35n6p2955.

VIEIRA, R. F.; PINTO, C. M. F.; VIEIRA, C. E. In: 101 culturas: manual de tecnologia agrícola. Paula Júnior, T. J. de. Venzon, M. Belo Horizonte: EPAMIG, 2007. 800p.

OLIVEIRA, F. N.; TORRES, S. B.; NOGUEIRA, N. W.; FREITAS, R. M. O. Viabilidade de sementes de pereiro-vermelho (Simira gardneriana MR Barbosa \& Peixoto) pelo teste de tetrazólio. Journal of Seed Science, v. 38, n. 1, 2016. http://dx.doi.org/10.5433/1679-0359.2014v35n6p2955.

PACHECO, M. V.; ARAÚJO, F. S.; FERRARI, C. S.; BRUNO, R. D. L. A. Germinação de sementes de Combretum leprosum Mart. Revista Caatinga, v. 27, n. 1, p. 154-162, 2014.

ZUCARELI, V.; FERREIRA, G.; AMARO, A. C. E.; ARAÚJO, F. P. D. Fotoperíodo, temperatura e reguladores vegetais na germinação de sementes de Passiflora cincinnata Mast. Revista Brasileira de Sementes, v. 31, n. 3, p. 106-114, 2009. http://dx.doi.org/10.1590/S0101-31222009000300012 